\title{
Kompetenzerwartungen in der Ausbildung Psychologischer Psychotherapeuten und Kinder- und Jugendtherapeuten in der Verhaltenstherapie
}

\author{
Zarbock, Gerhard ; Rodde, Sibyll ; Ströhm, Walter ; Schulz, Holger ; Watzke, Birgit
}

\begin{abstract}
Hintergrund: Die Psychotherapieausbildung wird mehr und mehr zum Gegenstand empirischer Forschung. In Deutschland hat sich die Psychotherapeutenausbildung nach Inkrafttreten des Psychotherapeutengesetzes erheblich verändert. In Bezug auf die neuen Ausbildungsgänge beginnt nun die Phase der empirischen Evaluation. Ziel der vorliegenden Studie war es, zu dieser Entwicklung im Hinblick auf die Ausbildung in der Verhaltenstherapie (VT) beizutragen. Methode: Eine Stichprobe von n = 2106 Ausbildungskandidaten in VT konnte mit einem Fragebogen zu Ausbildungsbedingungen, Ausbildungserfahrungen und subjektiven Kompetenzerwartungen in Bezug auf psychotherapeutische Kompetenzen befragt werden. Der Fragebogen wurde in Anlehnung an Instrumente zum Hochschulranking in einem mehrstufigen Prozess entwickelt. Durch hierarchische, mehrfach lineare Regressionsanalysen wurde versucht, 5 psychotherapeutische Kompetenzbereiche durch soziodemografische, ausbildungsbezogene und institutionsstrukturelle Prädiktoren vorherzusagen. Ergebnisse: Insgesamt war die erklärte Varianz gering. Ein als angemessen beurteiltes Aufwand/Nutzen-Verhältnis bei der Ausbildung und die persönliche Unterstützung durch einen kompetenten Supervisor konnten jedoch in allen untersuchten Kompetenzbereichen signifikant zur Erklärung der Varianz beitragen. Schlussfolgerungen: Um unterschiedliche Bedürfnisse von Ausbildungskandidaten zu berücksichtigen, sollten unterschiedliche Ausbildungsangebote gemacht werden. Persönliche Unterstützung in der Supervision durch einen erfahrenen Kollegen ist wesentlich für die Entwicklung einer positiven professionellen Kompetenzerwartung.
\end{abstract}

DOI: https://doi.org/10.1159/000335773

Posted at the Zurich Open Repository and Archive, University of Zurich

ZORA URL: https://doi.org/10.5167/uzh-107339

Journal Article

Published Version

Originally published at:

Zarbock, Gerhard; Rodde, Sibyll; Ströhm, Walter; Schulz, Holger; Watzke, Birgit (2012). Kompetenzerwartungen in der Ausbildung Psychologischer Psychotherapeuten und Kinder- und Jugendtherapeuten in der Verhaltenstherapie. Verhaltenstherapie, 22(1):3.

DOI: https://doi.org/10.1159/000335773 


\title{
Kompetenzerwartungen in der Ausbildung Psychologischer Psychotherapeuten und Kinder- und Jugendtherapeuten in der Verhaltenstherapie
}

\author{
Gerhard Zarbock $^{\mathrm{a}}$ Sibyll Rodde ${ }^{\mathrm{b}}$ Walter Ströhm ${ }^{\mathrm{c}}$ \\ Holger Schulz ${ }^{d}$ Birgit Watzke ${ }^{d}$ \\ a IVAH Institut für Verhaltenstherapie-Ausbildung Hamburg gGmbH, \\ ${ }^{\mathrm{b}}$ APV, Gesellschaft für Angewandte Psychologie und Verhaltensmedizin, Münster, \\ ${ }^{\mathrm{c}}$ DVT, Deutscher Fachverband für Verhaltenstherapie e.V., Münster, \\ ${ }^{d}$ Forschungsgruppe Psychotherapeutische Versorgung, Institut für Medizinische Psychologie, Universitätsklinikum Hamburg-Eppendorf, \\ Deutschland
}

\section{Schlüsselwörter}

Psychotherapieausbildung · Kompetenzerwartung ·

Professionalisierungsprozess · Ausbildungsforschung

\section{Zusammenfassung}

Hintergrund: Die Psychotherapieausbildung wird mehr und mehr zum Gegenstand empirischer Forschung. In Deutschland hat sich die Psychotherapeutenausbildung nach Inkrafttreten des Psychotherapeutengesetzes erheblich verändert. In Bezug auf die neuen Ausbildungsgänge beginnt nun die Phase der empirischen Evaluation. Ziel der vorliegenden Studie war es, zu dieser Entwicklung im Hinblick auf die Ausbildung in der Verhaltenstherapie (VT) beizutragen. Methode: Eine Stichprobe von $n=2106$ Ausbildungskandidaten in VT konnte mit einem Fragebogen zu Ausbildungsbedingungen, Ausbildungserfahrungen und subjektiven Kompetenzerwartungen in Bezug auf psychotherapeutische Kompetenzen befragt werden. Der Fragebogen wurde in Anlehnung an Instrumente zum Hochschulranking in einem mehrstufigen Prozess entwickelt. Durch hierarchische, mehrfach lineare Regressionsanalysen wurde versucht, 5 psychotherapeutische Kompetenzbereiche durch soziodemografische, ausbildungsbezogene und institutionsstrukturelle Prädiktoren vorherzusagen. Ergebnisse: Insgesamt war die erklärte Varianz gering. Ein als angemessen beurteiltes Aufwand/Nutzen-Verhältnis bei der Ausbildung und die persönliche Unterstützung durch einen kompetenten Supervisor konnten jedoch in allen untersuchten Kompetenzbereichen signifikant zur Erklärung der Varianz beitragen. Schlussfolgerungen: Um unterschiedliche Bedürfnisse von Ausbildungskandidaten zu berücksichtigen, sollten unterschiedliche Ausbildungsangebote gemacht werden. Persönliche Unterstützung in der Supervision durch einen erfahrenen Kollegen ist wesentlich für die Entwicklung einer positiven professionellen Kompetenzerwartung.

\section{Keywords}

Psychotherapy training - Competency expectations . Professionalization process - Training research

\section{Summary}

Competency Expectations in the Training of Psychotherapists Specializing in Cognitive Behavioral Therapy Background: Psychotherapy training is becoming more and more a topic for empirical research. In Germany, the training in psychotherapy underwent major changes through the establishment of a new law regulating the training in and the practice of psychotherapy. These new training curricula have just recently started to become a focus of research. The aim of this study was to contribute to this issue with regard to training in behavioral therapy (BT). Method: A sample of $n=2106$ trainees in BT filled out a questionnaire concerning conditions of training, experience during training, and self-estimated competence expectations. The questionnaire was constructed with reference to instruments for ranking universities and in a multiple-stage approach. Using hierarchical, multiple linear regression analysis, it was attempted to predict 5 competence domains of psychotherapy by sociodemographic, training-related and institution structure-related predictors. Results: Overall, the explained variance was small. A cost/performance ratio regarding the training judged as satisfactory and the personal support by a competent supervisor could, in all 5 competence domains, significantly explain the variance. Conclusions: Due to the different needs of trainees, different training options should be offered. The personal relationship with an experienced senior seems to be essential for the development of a positive expectancy of professional competence.

\section{KARGER \\ Fax +497614520714 \\ Information@Karger.de}

www.karger.com
(C) 2012 S. Karger GmbH, Freiburg 1016-6262/12/0221-0027\$38.00/0

Accessible online at: www.karger.com/ver
Dr. Gerhard Zarbock

IVAH Institut für Verhaltenstherapie-Ausbildung Hamburg gGmbH

Bachstraße 48, 22083 Hamburg, Deutschland

Tel. +49 40 22-1620, Fax -6669

info@ivah.de 


\section{Einleitung}

Die Ausbildung von Psychotherapeuten wird zunehmend zum Gegenstand der empirischen Forschung [Strauß und Kohl, 2009]. Obwohl experimentelle, randomisierte Forschungsstudien schwierig durchzuführen sind und viele Aspekte noch untersucht werden müssen, ist die Befundlage über die psychotherapeutische Ausbildung in Deutschland durchaus umfangreich [Lairaiter und Botermann, 2005]. Die letzte international groß angelegte, systematische Betrachtung des Themas liegt jedoch schon länger zurück. Das «Handbook of Psychotherapy and Behavior Change» widmete dem Thema zuletzt vor 23 Jahren (3. Auflage von 1986) ein Kapitel. In der 4. (1994) und 5. Auflage (2004) der Edition kommt das Thema der psychotherapeutischen Ausbildung nicht mehr vor.

Im Allgemeinen ruht die psychotherapeutische Ausbildung auf vier Säulen: 1. einer theoretischen und Techniken übenden Ausbildung, 2. der Behandlung von Patienten, 3. der Supervision der Auszubildenden und 4. der Selbsterfahrung und/oder der Selbsttherapie [Lairaiter und Botermann, 2005]. Bis jetzt zeigen die allgemeinen Ergebnisse bezüglich der Auswirkungen von spezifischen Ausbildungskomponenten auf den Erfolg der Ausbildung («become a good therapist»), dass die dem Kandidaten beigebrachten, spezifischen und praxisorientierten Fähigkeiten (z.B. Anwendung spezifischer Techniken, wie Expositionsverfahren mit Reaktionsverhinderung und verbale Validierungstechniken) positive Auswirkungen auf die Behandlung der Patienten während der Ausbildungszeit haben. Obwohl Selbsterfahrung und Selbsttherapie von den Kandidaten sehr geschätzt werden, konnte ein positiver Effekt dieser Ausbildungsbestandteile auf die durchgeführten Ausbildungstherapien empirisch bisher nicht explizit nachgewiesen werden.

In den verschiedenen psychotherapeutischen Verfahren (z.B. Psychoanalyse, humanistische Ansätze, Familientherapie und kognitive Verhaltenstherapie (KVT)) variieren die Anteile der einzelnen angebotenen Ausbildungskomponenten erheblich. Darüber hinaus werden die Ausbildungskomponenten in Bezug auf die Auswirkungen, die sie auf den Ausbildungserfolg haben, innerhalb der einzelnen Verfahren theoriegeleitet sehr unterschiedlich oder sogar diametral beurteilt [Lairaiter und Botermann, 2005]. Als Folge der Unterschiede in den psychotherapeutischen Theorien sollten die auf verschiedenen psychotherapeutischen Konzepten basierenden Ausbildungen auch getrennt untersucht werden, da die spezifischen Eigenschaften der verschiedenen Konzepte sonst verborgen bleiben könnten.

In Deutschland sind Verhaltenstherapie (VT)- und psychodynamische Therapieansätze die Hauptbehandlungsmethoden sowohl ambulanter als auch stationärer psychotherapeutischer Behandlung [Schulz et al., 2008]. Die Mehrheit der existierenden Evaluationen der VT-Ausbildungen im deutschsprachigen Raum ist älter als 10 Jahre. Darüber hinaus evaluierte eine Vielzahl dieser Studien Ausbildungen, die sich nicht in Übereinstimmung mit Richtlinien und Standards des deutschen Psychotherapeutengesetzes von 1999 befanden (PsychThG).
Eine Befragung von VT-Auszubildenden, die auf kleinen Stichproben einer einzigen Ausbildungsinstitution basierte, zeigte, dass verschiedene Ausbildungskomponenten für die KVT-Ausbildung als hilfreich beurteilt wurden. Zimmer und Zimmer [1998] befragten 75 Kandidaten einer staatlich akkreditierten VT-Ausbildung. Die befragten Kandidaten hielten besonders Selbsterfahrung und Supervision für hilfreich, um Beziehungen professionell gestalten zu lernen. Einzel- und Gruppenselbsterfahrung wurden gut beurteilt, da man sich selbst und seine eigenen Reaktionsmuster kennenlernen würde. Blockseminare mit einem hohen Anteil an Rollenspielen und Supervision wurden ebenfalls zum Erwerb therapeutischer Kompetenzen positiv bewertet. Insgesamt wurde das Einzelsetting in Supervision und Selbsterfahrung als besonders empfehlenswert beurteilt.

Schulz et al. [1998] evaluierten die Einschätzungen von Auszubildenden $(\mathrm{n}=45)$ einer KVT-Ausbildung mit einer durchschnittlichen Ausbildungszeit von 3,8 Jahren. Theorieseminare, Supervision und Selbstreflexion wurden durchweg positiv bewertet. Am Ende der Ausbildung hatten die Kandidaten Selbstvertrauen in ihre therapeutischen Fähigkeiten und entwickelten eine professionelle Identität. von Hopffgarten et al. [1998] befragten Kandidaten $(n=48)$ einer VT-Ausbildung von 6 Semestern Dauer. Die Kandidaten berichteten von einem signifikanten Kompetenzgewinn in psychotherapeutisch relevanten Bereichen [Grawe, 1994], wie z.B. Problemlösungskompetenz, Motivationsklärung, Problemaktivierung, Ressourcenaktivierung und therapeutische Beziehungen.

Scherer et al. [2005] analysierten die ersten Ergebnisse der letzten Approbationsprüfungen im Bereich Behandlungskonzepte (z.B. psychodynamische Psychotherapie, Psychoanalyse, KVT) in Deutschland nach Einführung des neuen Psychotherapiegesetzes im Zeitraum von Herbst 2002 bis Frühling 2005. Sie fanden, dass die Ergebnisse des mündlichen Teils des Examens deutlich besser waren als die des schriftlichen Teils. Im Vergleich zu den jüngeren Auszubildenden erreichten die Älteren weniger Punkte in der schriftlichen Prüfung, und Kandidaten in Vollzeitausbildung schnitten besser $a b$ als jene in Teilzeit. Die Auswertung ergab auch, dass Kinder- und Jugendpsychotherapeuten (KJP) schlechter abschnitten als Erwachsenenpsychotherapeuten. Es konnte auch gezeigt werden, dass die letzte Examensabschlussnote des ersten Universitätsabschlusses (z.B. Psychologie, Pädagogik, Sozialpädagogik) einen deutlichen Effekt auf die Varianz der Ergebnisse des schriftlichen Examens für beide Berufsgruppen hatte.

Auf der Grundlage einer Online-Studie sammelte von Hölzel [2006] Daten über die finanzielle Situation von Auszubildenden während ihrer Ausbildung. Die Studie konnte zeigen, dass die Auszubildenden während der Ausbildung mit großen finanziellen Belastungen zu kämpfen haben $(n=444)$. Über $50 \%$ der an der Studie Teilnehmenden erhielten für ihre Arbeit in einer psychiatrischen Klinik (mindestens 1200 Stunden, mindestens ein Jahr), die gemäß der Ausbildungs- 
und Prüfungsverordnung erforderlich ist, keinen Lohn. Ein großer Prozentsatz musste sich von ihren Partnern oder Eltern finanziell abhängig machen, um ihre Ausbildung erfolgreich abschließen zu können.

Will man die Qualität der Ausbildung beurteilen, ist die Einstellung der Auszubildenden nicht nur im Hinblick auf den Aspekt der Qualitätskontrolle [Sonntag et al., 2009; Strauß und Freyberger, 2009], sondern vor allem in Bezug auf ihre zukünftige Weiterentwicklung als Psychotherapeuten relevant. Da die Auszubildenden positive Erfahrungen während ihrer Ausbildung sammeln können - z.B. zufriedenstellende Erstkontakte zu Patienten, Erwerb von klinischem Wissen und Erfahrung -, kann man annehmen, dass der Zuwachs an beruflicher Erfahrung rückblickend zu einer allgemeinen positiven Beurteilung der eigenen Karriere führen dürfte. Die ersten Kompetenzerfahrungen am Anfang der Ausbildung bilden die Basis für eine positive Berufsidentität. Laut Orlinskys und Roennestads [2005] Modell der therapeutischen Arbeit und beruflichen Entwicklung von Psychotherapeuten kann angenommen werden, dass vom Therapeuten erlebtes «Healing Involvement» (heilende Involvierung) mit Patienten und «Currently Experienced Growth» (aktuell erlebtes professionelles Wachstum) im Laufe gelingender beruflicher Sozialisation anwachsen werden. Bei den beiden Variablen handelt es sich um «die» zwei maßgeblichen Variablen der professionellen Entwicklung von Psychotherapeuten. Empirische Beweise, die die Relation zwischen den oben genannten Variablen stützen, können bei Willutzki et al. [1997] gefunden werden. Die Autoren untersuchten deutsche und Schweizer Psychotherapeuten $(\mathrm{n}=1065)$ und fanden heraus, dass das professionelle Selbstvertrauen von Psychotherapeuten anhand ihrer guten Erfahrungen in Kursen und Seminaren während der Ausbildung vorhergesagt werden kann.

Vor diesem Hintergrund war das Ziel dieser Studie, die aktuellen professionellen Kompetenzerwartungen von angehenden, sich bereits in der VT-Ausbildung befindenden Psychotherapeuten zu untersuchen. Die Stichprobe umfasste Auszubildende aus verschiedenen Ausbildungszentren des Deutschen Fachverbands für Verhaltenstherapie (DVT). Untersucht werden sollte, ob und welche Variablen die professionellen Kompetenzerwartungen vorhersagen können. Das Konstrukt der Kompetenzerwartungen dürfte für die weitere berufliche Entwicklung von zentraler Bedeutung sein, da positive Kompetenzerwartungen die Grundlage dafür sind, therapeutische Kontakte als «Healing Involvement» [Orlinsky und Roennestedt, 2005] erfahren zu können und «Currently Experienced Growth» zu erleben.

\section{Teilnehmer und Methode}

\section{Teilnehmer}

Wir befragten Auszubildende, die gerade an einem VT-Ausbildungscurriculum teilnahmen, um Psychologische Psychotherapeuten (PP) oder KJP zu werden. Die Ausbildung in Deutschland wird vom Psychothera- piegesetz bestimmt, dauert 3 (Vollzeit) bis 5 Jahre (Teilzeit) und umfasst mindestens 4220 Ausbildungsstunden. Die Ausbildung wird entweder als Postgraduierten-Kurs von einer Universität oder von einer staatlich lizensierten Privatinstitution angeboten, die den Universitäten gleichrangig sind.

Um Qualitätsstandards zu sichern, haben sich mehrere der wichtigsten Privatinstitutionen im DVT zusammengeschlossen und bilden zusammen mehr als 50\% der angehenden Psychotherapeuten im Bereich VT aus.

\section{Befragungsablauf}

Im Juni 2008 wurden an 33 DVT-Instituten Umfragen durchgeführt: Jedes Institut bekam 4 Fragebögen mit Briefumschlägen und Rückporto zugestellt, einen Fragebogen für den Institutsleiter und 3 SupervisorenFragebögen. Die Institutsleiter wurden in einem Anschreiben gebeten, die Supervisoren-Fragebögen an 3 Ausbilder ihrer Wahl zu verteilen. Der Rücklauf der Fragebögen an ein Mitglied der Expertengruppe des DVT war Ende August 2008 abgeschlossen.

Um die Anonymität in der Befragung der Ausbilder zu gewährleisten, war jeder Fragebogen mit einem Institutscode gekennzeichnet. Gleichzeitig wurde den Ausbildern ein individueller Code für die Rückantwort gegeben. Mithilfe dieses individuellen Rückcodes war es möglich herauszufinden, ob Ausbilder mehr als eine Ausbildungsstätte bewerteten. Da wir nur 78 individuelle Rückcodes zurückbekamen, müssen wir davon ausgehen, dass Ausbilder im Allgemeinen nur eine Ausbildungsinstitution bewertet haben.

Im Sommer 2008 wurden dann Fragebögen für Ausbildungskandidaten in Zusammenarbeit mit den Institutsleitern und unter Berücksichtigung ihrer Ideen und Kommentare entworfen. Ende Oktober 2008 bekamen alle 33 Ausbildungsinstitutionen ihre Ausbildungskandidaten-Fragebögen, inklusive Briefumschläge und Rückporto. Im Anschreiben wurden die Institutsleiter gebeten, die Fragebögen in den Seminaren zu verteilen. Die Organisation der Verteilung oblag den Institutionen selbst. Die Befragung der Auszubildenden erfolgte anonym. Nach Abschluss der Befragung sammelte jeweils ein Auszubildender im Seminar die Fragebögen ein und sandte diese in dem dafür vorgesehenen Umschlag postalisch an die Expertengruppe. Einige der Fragebögen wurden von den Auszubildenden einzeln zurückgeschickt. Bis Januar 2009 war die Datensammlung vollständig abgeschlossen.

\section{Messinstrument}

Mit dem Ziel, die Qualität der Psychotherapie-Ausbildung zu sichern und zu verbessern, wurden innerhalb der DVT-Institute bereits standardisierte Feedback-Fragebögen zur Qualität der verschiedenen Seminare eingesetzt.

Innerhalb des DVT kam der Wunsch auf, eine umfangreiche Evaluationsstudie durchzuführen, die sowohl die organisatorischen, strukturellen und inhaltlichen Aspekte der Psychotherapie-Ausbildung an jedem Mitgliedsinstitut bewertet als auch die Qualität der einzelnen Ausbildungsbestandteile. In Kooperation mit dem DVT-Präsidenten entwickelte eine Gruppe von Experten in einem 2-stufigen Prozess einen Fragebogen für Ausbildungskandidaten. Im ersten Schritt wurde den Institutsleitern und Ausbildern ein spezifischer Fragebogen (siehe oben) gegeben. Ihre Antworten wurden bei der Konzeptionierung des Auszubildenden-Fragebogens berücksichtigt. Die Expertengruppe war zusammengesetzt aus dem DVT-Präsidenten und 3 anderen Mitgliedern mit ökonomischem, psychologischem und VT-beruflichem Hintergrund; alle wiesen vielfältige Forschungserfahrungen auf.

Gemäß den Prinzipien des CHE-Hochschulrankings [Berghoff et al., 2007] (CHE = Centrum für Hochschulentwicklung) sammelten die Institutsleiter mittels Fragebögen Basisdaten zu den verschiedenen teilnehmenden Instituten, z.B. Anzahl der Dozenten und sonstigen Angestellten, Anzahl der Auszubildenden in Voll- und Teilzeit, zur Verfügung stehendes Material und technische Ausrüstung der Institutionen. Zusätzlich wurde eine gekürzte Fassung des Fragebogens für die Betreuer entwickelt. 
Der Fragebogen für die Auszubildenden umfasste 152 Items. 9 davon erfragten demografische Daten. 46 Items erhoben detaillierte Informationen zur Psychotherapie-Ausbildung, wie z.B. den Ausbildungsstart, Volloder Teilzeit-Beschäftigung, PP- oder KJP-Ausbildung, aktuellen Ausbildungsstand, voraussichtliches Ausbildungsende, Gründe für mögliche Verzögerungen, eventuelle Institutswechsel oder Ausbildungsabbrüche, Abschluss des psychiatrischen Praktikums, Ausbildungskosten, Einkommen und Finanzierung der Ausbildung. Entsprechende Antwortoptionen wurden zur Verfügung gestellt. Weiterhin beurteilten die Ausbildungskandidaten ihre Ausbildungsstätte nach der Qualität der Räumlichkeiten, Infrastruktur, Organisation, Kosten und Ansprechpartnern (17 Items). Mit 75 Items wurden verschiedene Aspekte der Ausbildung abgedeckt: Die Auszubildenden bewerteten ihre Zufriedenheit mit 1. den theoretischen Seminaren, 2. der praktischen Tätigkeit (psychiatrisches und psychotherapeutisches Praktikum), 3. der Selbsterfahrung, 4. der praktischen Ausbildung (eigene Fallbehandlung), 5. der Supervision und 6. der Vorbereitung des Staatsexamens, inklusive der organisatorischen Vorbereitungen. Schließlich beurteilten sie ihre therapeutische Qualität am Ende der Ausbildung (5 Items). Für alle Items verwendeten wir eine 6-PunkteLikert-Skala, die von $1=$ sehr gut bis $6=$ sehr schlecht reichte, ergänzt durch die Option «nicht zutreffend».

Das Ausbildungsergebnis wurde durch Fragen zu den Kompetenzerwartungen der Auszubildenden operationalisiert. Dafür wurden Fragen wie «Wie beurteilen Sie Ihre therapeutischen Kompetenzen am Ende der Ausbildung?» auf der 6-Punkte-Likert-Skala von 1 bis 6 (siehe oben) für folgende Bereiche gestellt: 1. Kenntnisse über Symptome und Ätiologie psychischer Störungen, 2. prozessuale Fähigkeiten (Diagnostik, Therapieplanung, Intervention), 3. interpersonelle Kompetenzen (therapeutische Beziehungen, Fähigkeiten im Umgang mit «schwierigen Patienten» usw.), 4. Formalitäten (Krankenversicherungsanträge, Berichte, Aktenführung), 5. Selbsterfahrung.

In Bezug auf die Ausbildungskandidaten wurden folgende Informationen zu Ausbildung in Ergänzung mit soziodemografischen Daten aufgenommen: PP- und/oder KJP-Ausbildung; Voll- oder Teilzeit; aktueller Stand und geschätzte Dauer der Ausbildung; Anteil zusätzlicher Erwerbsarbeit, um die Ausbildung zu finanzieren; Stand des Praktikums (1., 2. oder 3. Phase des Praktikums). Weiterhin bewerteten die Auszubildenden die Wichtigkeit des Rufes der Ausbildungsstätte und/oder berufliche Gründe für ihre Auswahl der Ausbildungsstätte (6-Punkte-LikertSkala, 1 = sehr wichtig bis $6=$ unwichtig).

Zusätzlich bewerteten die Auszubildenden institutionsbezogene Parameter (strukturell-prozessuale Parameter) auf einer 6-Punkte-LikertSkala von $1=$ sehr gut bis $6=$ sehr schlecht. Diese Parameter umfassten die Organisation der Ausbildung, das Kosten/Leistungs-Verhältnis der Ausbildungsoptionen, Beratung und Betreuung/Begleitung durch den Institutsleiter bis zum Abschluss der Ausbildung, Unterstützung bei Schwierigkeiten während der Ausbildung, Berücksichtigung der Bedürfnisse der Auszubildenden, Offenheit für Feedback von den Auszubildenden, Integration und Weiterentwicklung der Evaluierungsergebnisse in zukünftigen Seminaren. Im Hinblick auf die Betreuung bewerteten die Auszubildenden die pädagogische Qualifikation und die didaktischen Fähigkeiten ihrer Ausbilder, die Angemessenheit der Supervisionsintensität und die persönliche Unterstützung durch die Ausbilder bei der Professionalisierung therapeutischen Verhaltens. Die Auszubildenden bewerteten auch die Größe der Ausbildungsgruppen (Anzahl Auszubildender pro Gruppe). Darüber hinaus wurden sie gebeten, Informationen zu den Gesamtkosten der Ausbildung zur Verfügung zu stellen.

\section{Statistische Analyse und Evaluierungsstrategien}

$\mathrm{t}$-Tests für metrische und $\chi^{2}$-Tests für kategorische Variablen wurden durchgeführt, um soziodemografische und ausbildungsbezogene Attribute für PP- und KJP-Auszubildende miteinander zu vergleichen. Cohens [1998] vorgeschlagene Klassifikation von kleinen $(d \geq 0,20 ; \phi \geq$ $0,10)$, mittleren $(\mathrm{d} \geq 0,50 ; \phi \geq 0,30)$ und großen $(\mathrm{d} \geq 0,80 ; \phi \geq 0,50)$ Aus- wirkungen wurde angewendet, um das Ausmaß der Effekte abzuschätzen. Es wurden nur Differenzen von mindestens kleiner Effektstärke mit einbezogen.

Es wurden hierarchische, mehrfach lineare Regressionen berechnet, um 5 Bereiche bezüglich der Kompetenzerwartungen vorherzusagen (Kompetenz in Symptomkenntnissen und Ätiologie, Prozessdiagnostik und therapeutische Fähigkeiten, interpersonelle Fähigkeiten und Umgang mit formellen Erfordernissen (z.B. Umgang mit Krankenversicherungsunternehmen, Berichterstattung, Selbsterfahrung). Die Auswahl der zu untersuchenden Prädiktoren basierte auf inhaltlichen und theoretischen Aspekten. Block 1 der Regression umfasste soziodemografische Variablen, Block 2 umfasste ausbildungsbezogene Attribute und Block 3 bestand aus strukturell-prozessualen Parametern der Ausbildungsstätte (siehe Tab. 3, Sektion Vergleiche/Maßnahmen, für einzelne Prädiktoren). Für jedes Regressionsmodell wurde sowohl der Anteil der erklärten Varianz an der Gesamtvarianz als auch die graduell erklärte Varianz von Block 2 und 3 festgelegt sowie die Abschätzung/Einschätzung des relativen Beitrags der einzelnen Prädiktoren mittels angepasster BetaGewichte.

\section{Teilnehmer}

Von den 34 vereinigten DVT-Ausbildungsinstitutionen nahmen 33 an der Studie teil. Von 33 befragten Institutsleitern sendeten 32 ihre Fragebögen zurück. Daneben bewerteten 78 Ausbilder die Ausbildungsstätten anonym. Alter und Geschlecht beider Gruppen wurden nicht aufgenommen.

Laut DVT befanden sich zum Zeitpunkt der Studie insgesamt 3664 Kandidaten in der Ausbildung an den 33 Ausbildungsstätten. Jeder Institution sind ausreichend Fragebögen für die Auszubildenden zur Verfügung gestellt worden. 2106 Auszubildende (85,4\% weiblich, 14,6\% männlich) sandten ihre Fragebögen zurück; die Rücklaufquote betrug $57,5 \%$.

\section{Ergebnisse}

\section{Teilnehmer}

Tabelle 1 bietet einen Überblick über die soziodemografischen und ausbildungsbezogenen Variablen des einbezogenen Beispiels: Ein Großteil der Auszubildenden war weiblich (86,2\%); das Durchschnittsalter war 32,9 Jahre (Standardabweichung $(\mathrm{SD})=5,6$ ) weniger als zwei Drittel waren verheiratet oder lebten in Beziehungen. Die durchschnittliche Dauer der Ausbildung betrug 35,6 Monate (SD = 15,6), und mehr als die Hälfte der Auszubildenden (54,2\%) war in Vollzeitausbildung. Nur $10 \%$ planten, die Ausbildung nach 3 Jahren abzuschließen; ein Drittel der Auszubildenden plante, die Ausbildung in 4-5 Jahren zu beenden.

Wie erwartet, unterschieden sich die Auszubildenden in der Erwachsenen-Psychotherapie (PP) von den Auszubildenden in der Kinder- und Jugendpsychotherapie (KJP) hinsichtlich ihrer Universitätsabschlüsse. In Deutschland ist für eine Ausbildung zum Erwachsenen-Psychotherapeuten ein Universitätsabschluss im Fach Psychologie Voraussetzung; für eine Ausbildung zum Kinder- und Jugendpsychotherapeuten qualifiziert dagegen auch ein pädagogischer oder sozialpädagogischer Hochschulabschluss. Demnach hatte etwa die Hälfte der KJP-Auszubildenden zuvor Psychologie studiert, $36 \%$ hatten einen pädagogischen Hintergrund. Weitere signifikante Unterschiede - zumindest im Bereich kleiner Effekt- 
Tab. 1. Soziodemografische und ausbildungsbezogene Parameter der Ausbildungskandidaten, differenziert nach Art der Ausbildung

\begin{tabular}{|c|c|c|c|c|}
\hline Parameter & Summe & PP-Ausbildung & KJP-Ausbildung & $\begin{array}{l}\text { Vergleich zwischen PP- und KJP-Ausbildung, } \\
\text { ANOVA } / \chi^{2}\end{array}$ \\
\hline Mittleres Alter (SD), Jahre & $32,9(5,6)$ & $32,8(5,5)$ & $33,0(6,1)$ & $\mathrm{t}(1116)=0,569 ; \mathrm{p}=0,569 ; \mathrm{d}=0,04$ \\
\hline Geschlecht, \% & & & & $\chi^{2}(1, \mathrm{n}=1133)=0,008 ; \mathrm{p}=0,930 ; \phi=0,003$ \\
\hline Weiblich & 86,2 & 86,3 & 86,1 & \\
\hline Männlich & 13,8 & 13,7 & 13,9 & \\
\hline Familienstand, \% & & & & $\chi^{2}(3, \mathrm{n}=1127)=9,317 ; \mathrm{p}=0,025 ; \phi=0,091$ \\
\hline Ledig & 26,3 & 23,9 & 32,6 & \\
\hline In fester Partnerschaft lebend & 39,0 & 40,7 & 34,5 & \\
\hline Verheiratet & 32,1 & 32,9 & 29,9 & \\
\hline Geschieden/verwitwet & 2,6 & 2,4 & 3,0 & \\
\hline Kinder, \% & & & & $\chi^{2}(1, \mathrm{n}=1131)=1,087 ; \mathrm{p}=0,297 ; \phi=0,031$ \\
\hline Mit Kindern & 31,3 & 30,4 & 30,4 & \\
\hline Ohne Kinder & 68,7 & 69,6 & 69,6 & \\
\hline Abschluss, \% & & & & $\chi^{2}(3, \mathrm{n}=1140)=451,84 ; \mathrm{p}<0,001 ; \phi=0,630$ \\
\hline Psychologie & 86,6 & 99,6 & 51,6 & \\
\hline Pädagogik & 5,0 & 0,1 & 18,1 & \\
\hline Sozialpädagogik & 5,0 & 0,0 & 18,4 & \\
\hline Andere $^{\mathrm{a}}$ & 3,4 & 0,2 & 12,0 & \\
\hline Vollzeit/Teilzeit, \% & & & & $\chi^{2}(1, n=1133)=6,136 ; p=0,013 ; \phi=0,074$ \\
\hline Vollzeit & 54,2 & 56,4 & 48,2 & \\
\hline Teilzeit & 45,8 & 43,6 & 51,8 & \\
\hline $\begin{array}{l}\text { Mittlere Dauer der Ausbildung (SD), } \\
\text { Monate }\end{array}$ & $35,6(15,6)$ & $36,4(16,0)$ & $33,4(14,1)$ & $\mathrm{t}(1149)=2,911 ; \mathrm{p}=0,004, \mathrm{~d}=0,20$ \\
\hline $\begin{array}{l}\text { Geschätzte Ausdauer der } \\
\text { Ausbildung, \% }\end{array}$ & & & & $\chi^{2}(4, \mathrm{n}=1097)=6,597 ; \mathrm{p}<0,159 ; \phi=0,078$ \\
\hline 3 Jahre & 10,8 & 11,3 & 9,4 & \\
\hline 4 Jahre & 35,7 & 37,5 & 30,9 & \\
\hline 5 Jahre & 33,5 & 32,3 & 36,3 & \\
\hline 6 Jahre & 14,4 & 13,6 & 16,4 & \\
\hline 7 Jahre & 5,7 & 5,3 & 6,7 & \\
\hline
\end{tabular}

PP = Psychologischer Psychotherapeut, KJP = Kinder- und Jugendpsychotherapeut, SD = Standardabweichung.

${ }^{a}$ Pädagogik, Sozialarbeit, Sonderpädagogik, Heilpädagogik, Musiktherapie, Kunsttherapie, Medizin. Mehrfachantworten waren möglich.

stärken - fanden sich in Bezug auf die Ausbildungsdauer (KJP-Ausbildungskandidaten waren durchschnittlich 3 Monate weniger in Ausbildung). Bei allen anderen Variablen gab es keine signifikanten Unterschiede.

\section{Kompetenzerwartungen der Ausbildungskandidaten}

Die Bedeutung und Standardabweichung der Kompetenzerwartungen sind in Tabelle 2 dargestellt. Im Durchschnitt wurde die Mehrheit der 5 Kompetenzerwartungsbereiche hoch bis sehr hoch bewertet. Hohe Bewertungen wie diese findet man auch in der Forschung zu Patienten- und Kundenzufriedenheit in der Gesundheitsvorsorge [z.B. Williams et al., 1998]. Demnach bieten die Varianzen und Häufigkeitsverteilung der Variablen eine zusätzliche Information. Bezüglich des Inhalts war die Varianz in allen 5 Kompetenzbereichen von Bedeutung. Einerseits beurteilte ein wesentlicher Anteil der Auszubildenden ihre Kompetenzerwartungen in einem oder mehreren der 5 Bereiche als «sehr gut» (jeweils 258-281 Auszubildende); andererseits bewerteten 157-273 Auszubildende die Bereiche als «zufriedenstellend».

Signifikante Unterschiede - mit kleinen Effektstärken zeigten sich zwischen den PP- und KJP-Auszubildenden bei deren Kompetenzerwartung hinsichtlich «Symptome und Ätiologie» sowie «interpersoneller Fähigkeiten». Frauen und Männer unterschieden sich nicht systematisch voneinander bezüglich ihrer Kompetenzerwartung. Zwar erreicht die interpersonelle Kompetenzerwartung aufgrund der hohen Stichprobengröße tendenzielle Signifikanz zwischen Frauen und Männern, der Unterschied ist aber noch nicht einmal von kleiner Effektstärke und somit vernachlässigbar. Die Korrelation zwischen den 5 Punkten der Kompetenzerwartung reichte von $0,31 \leq \mathrm{r} \leq 0,59$ (Tab. 2 ). 
Tab. 2. Kompetenzerwartungen differenziert nach Frauen und Männern sowie nach Art der Ausbildung

\begin{tabular}{|c|c|c|c|c|c|c|c|c|c|c|}
\hline & \multicolumn{10}{|c|}{ Kompetenzerwartungen } \\
\hline & \multicolumn{2}{|c|}{$\begin{array}{l}\text { 1. Symptomkenntnisse, } \\
\mathrm{n}=1155\end{array}$} & \multicolumn{2}{|c|}{$\begin{array}{l}\text { 2. Prozessuale } \\
\text { Fähigkeiten, } n=1156\end{array}$} & \multicolumn{2}{|c|}{$\begin{array}{l}\text { 3. Interpersersonelle } \\
\text { Kompetenz, } \mathrm{n}=1148\end{array}$} & \multicolumn{2}{|c|}{$\begin{array}{l}\text { 4. Formalitäten, } \\
\mathrm{n}=1139\end{array}$} & \multicolumn{2}{|c|}{$\begin{array}{l}\text { 5. Selbsterfahrung, } \\
\mathrm{n}=1144\end{array}$} \\
\hline & M & $\mathrm{SD}$ & M & $\mathrm{SD}$ & M & $\mathrm{SD}$ & M & $\mathrm{SD}$ & M & $\mathrm{SD}$ \\
\hline Gesamt & 1,93 & 0,63 & 1,92 & 0,66 & 2,05 & 0,78 & 2,03 & 0,78 & 2,00 & 0,75 \\
\hline \multicolumn{11}{|l|}{ Geschlecht } \\
\hline Frauen $(\mathrm{n}=982)$ & 1,93 & 0,63 & 1,91 & 0,66 & 2,07 & 0,78 & 2,02 & 0,78 & 1,99 & 0,73 \\
\hline Männer (n = 157) & 1,93 & 0,67 & 1,97 & 0,69 & 1,94 & 0,81 & 2,05 & 0,82 & 2,05 & 0,83 \\
\hline $\begin{array}{l}\text { Statistischer Vergleich } \\
\text { Frauen/Männer }\end{array}$ & \multicolumn{2}{|c|}{$\begin{array}{l}\mathrm{t}(1137)=0,04 \\
\mathrm{p}=0,965 ; \mathrm{d}=0,0\end{array}$} & \multicolumn{2}{|c|}{$\begin{array}{l}\mathrm{t}(1138)=-0,96 \\
\mathrm{p}=0,338 ; \mathrm{d}=0,08\end{array}$} & \multicolumn{2}{|c|}{$\begin{array}{l}\mathrm{t}(1130)=1,96 \\
\mathrm{p}=0,051 ; \mathrm{d}=0,16\end{array}$} & \multicolumn{2}{|c|}{$\begin{array}{l}\mathrm{t}(1121)=0,46 \\
\mathrm{p}=0,643 ; \mathrm{d}=0,04\end{array}$} & \multicolumn{2}{|c|}{$\begin{array}{l}\mathrm{t}(1126)=1,00 \\
\mathrm{p}=0,315 ; \mathrm{d}=0,08\end{array}$} \\
\hline \multicolumn{11}{|l|}{ Art der Ausbildung } \\
\hline $\mathrm{PP}(\mathrm{n}=835)$ & 1,89 & 0,63 & 1,93 & 0,66 & 2,01 & 0,76 & 2,01 & 0,78 & 1,98 & 0,75 \\
\hline $\mathrm{KJP}(\mathrm{n}=314)$ & 2,03 & 0,64 & 1,88 & 0,64 & 2,15 & 0,81 & 2,06 & 0,81 & 2,04 & 0,74 \\
\hline $\begin{array}{l}\text { Statistischer Vergleich } \\
\text { PP/KJP }\end{array}$ & \multicolumn{2}{|c|}{$\begin{array}{l}\mathrm{t}(1149)=3,46 \\
\mathrm{p}=0,001 ; \mathrm{d}=0,22\end{array}$} & \multicolumn{2}{|c|}{$\begin{array}{l}\mathrm{t}(1149)=-1,07 \\
\mathrm{p}=0,286 ; \mathrm{d}=0,08\end{array}$} & \multicolumn{2}{|c|}{$\begin{array}{l}\mathrm{t}(1149)=2,71 \\
\mathrm{p}=0,007 ; \mathrm{d}=0,25\end{array}$} & \multicolumn{2}{|c|}{$\begin{array}{l}\mathrm{t}(1149)=0,97 \\
\mathrm{p}=0,334 ; \mathrm{d}=0,060\end{array}$} & \multicolumn{2}{|c|}{$\begin{array}{l}\mathrm{t}(1149)=0,87 \\
\mathrm{p}=0,384 ; \mathrm{d}=0,06\end{array}$} \\
\hline \multicolumn{11}{|c|}{ Interkorrelationen Kompetenzerwartungen } \\
\hline $\begin{array}{l}\text { Prozessuale } \\
\text { Fähigkeiten }\end{array}$ & \multicolumn{2}{|c|}{$0,59 * *$} & & & & & & & & \\
\hline $\begin{array}{l}\text { Interpersersonelle } \\
\text { Kompetenz }\end{array}$ & \multicolumn{2}{|c|}{$0,36^{* *}$} & \multicolumn{2}{|c|}{$0,44 * *$} & & & & & & \\
\hline Formalitäten & \multicolumn{2}{|c|}{$0,33 * *$} & \multicolumn{2}{|c|}{$0,39 * *$} & \multicolumn{2}{|c|}{$0,33 * *$} & & & & \\
\hline Selbsterfahrung & \multicolumn{2}{|c|}{$0,31 * *$} & \multicolumn{2}{|c|}{$0,38 * *$} & $0,51 *$ & & \multicolumn{2}{|c|}{$0,36 * *$} & & \\
\hline \multicolumn{11}{|c|}{$\begin{array}{l}\mathrm{PP}=\text { Psychologischer Psychotherapeut, KJP }=\text { Kinder- und Jugendpsychotherapeut, } \mathrm{M}=\text { Mittelwert, } \mathrm{SD}=\mathrm{Standardabweichung} \text {. } \\
\text { Für die Bewertung der Kompetenzerwartungen wurde eine 6-Punkte-Skala von } 1=\text { sehr gut bis } 6=\text { sehr schlecht benutzt. } \\
\text { Variationen in der Stichprobengröße sind auf fehlende Werte bei einzelnen Variablen zurückzuführen. } \\
* * \text { Die Korrelation ist signifikant mit } \mathrm{p}<0,01 .\end{array}$} \\
\hline
\end{tabular}

Vorhersage von Kompetenzerwartungen durch Ausbildungskandidaten-, Ausbildungs- und institutionelle Variablen

Tabelle 3 beinhaltet die Mittelwerte und Standardabweichungen der Prädiktoren, die im Regressionsmodell einbezogen wurden.

Die Prädiktoren, die in die Regressionsanalyse in 3 Stufen einbezogen wurden, können 11,9\% $(\mathrm{F}(23$ 1135) $=7,81$; $\mathrm{p}<$ 0,001) der Varianz der Kriterien Kompetenzerwartung «Wissen von Symptomen/Ätiologie» erklären. Zusätzlich zu Block 1 (soziodemografische Parameter) trägt Block 2 (Ausbildungskandidaten-bezogene Parameter und allgemeine Bedingungen) zu einer signifikant höheren Erklärung der Varianz bei (incremental $R^{2}=0,037 ; F_{\text {Change }}(7,1149)=6,28$; $\mathrm{p}<0,001)$. Die zusätzlich erklärte Varianz der Prädiktoren von Block 3 (strukturell-prozessuale Parameter der Institutionen) ist ebenfalls signifikant (incremental $R^{2}=0,037 ; F_{\text {Change }}$ $(7,1149)=6,28 ; \mathrm{p}<0,001)$.

Für das Kriterium Kompetenzerwartung «prozessuale Fähigkeiten» erklärt das Modell 12,3\% der Gesamtvarianz $(\mathrm{F}(23$ 1135) $=8,05 ; \mathrm{p}<0,001)$. Zusätzlich zu Block 1 (soziodemografische Parameter) trägt Block 2 (Ausbildungskandidaten-bezogene Parameter und allgemeine Bedingungen) zu einer signifikant höheren Erklärung der Varianz bei (incremental $\left.R^{2}=0,047 ; F_{\text {Change }}(7,1149)=8,07 ; p<0,001\right)$. Die erklärte Varianz der Prädiktoren von Block 3 (strukturell-prozessuale Parameter der Institutionen) ist ebenfalls signifikant (incremental $\left.\mathrm{R}^{2}=0,089 ; \mathrm{F}_{\text {Change }}(14,1135)=8,43 ; \mathrm{p}<0,001\right)$.

Die Varianz des Kriteriums Kompetenzerwartung «interpersonelle Fähigkeiten» beträgt 8,3\% $(\mathrm{F}(23$ 1135) = 5,53; $\mathrm{p}<$ $0,001)$. Es gibt auch eine zusätzliche erklärte Varianz aufgrund von Block 2 (incremental $\mathrm{R}^{2}=0,032 ; \mathrm{F}_{\text {Change }}(7,1149)=$ 5,$49 ; \mathrm{p}<0,001$ ) und Block 3 (incremental $\mathrm{R}^{2}=0,064 ; \mathrm{F}_{\text {Change }}$ $(14,1135)=5,78 ; \mathrm{p}<0,001)$.

Die Varianz des Modells für Kompetenzerwartung «Formalitäten» beträgt 8,4\% $(\mathrm{F}(231135)=5,62 ; \mathrm{p}<0,001)$. Wieder gibt es eine erklärte Varianz aufgrund von Block 2 (incremental $\left.\mathrm{R}^{2}=0,023 ; \mathrm{F}_{\text {Change }}(7,1149)=3,79 ; \mathrm{p}<0,001\right)$ und Block 3 (incremental $\left.\mathrm{R}^{2}=0,077 ; \mathrm{F}_{\text {Change }}(14,1135)=6,95 ; \mathrm{p}<0,001\right)$.

Für das Kriterium Kompetenzerwartung «Selbsterfahrung» zeigt das Modell 9,1\% der Varianz $(\mathrm{F}(23$ 1135) = 6,03; $\mathrm{p}<0,001)$. Weiterhin gibt es eine erklärte Varianz aufgrund von Block 2 (incremental $\mathrm{R}^{2}=0,035 ; \mathrm{F}_{\text {Change }}(7,1149)=5,98$; $\mathrm{p}<0,001$ ) und Block 3 (incremental $\mathrm{R}^{2}=0,072 ; \mathrm{F}_{\text {Change }}$ (14, $1135)=6,55 ; \mathrm{p}<0,001)$. 
Tab. 3. Prädiktoren der Kompetenzerwartungen: soziodemografisch, ausbildungsbezogene und prozessuale Parameter

\begin{tabular}{|c|c|c|}
\hline Prädiktoren des geprüften Modells & $\mathrm{M}(\mathrm{SD}), \%$ & $\mathrm{n}$ \\
\hline \multicolumn{3}{|l|}{ Gruppe 1: soziodemografische Parameter } \\
\hline \multicolumn{3}{|l|}{ Alter $^{\mathrm{a}}$} \\
\hline \multicolumn{3}{|l|}{ Geschlecht ${ }^{\mathrm{a}}$} \\
\hline \multicolumn{3}{|l|}{ Gruppe 2: ausbildungsbezogene Parameter, z.B. allgemeine Ausbildungsbedingungen } \\
\hline \multicolumn{3}{|l|}{ Art der Ausbildung: PP/KJP } \\
\hline \multicolumn{3}{|l|}{ Art der Ausbildung: Volllzeit/Teilzeit ${ }^{\mathrm{a}}$} \\
\hline \multicolumn{2}{|l|}{ Aktueller Ausbildungsstand } & geschätzte Dauer der Ausbildung \\
\hline Anteil der zusätzlichen Arbeit zur Finanzierung der Ausbildung & $40,70(36,12)$ & 1148 \\
\hline Praktikum während des ersten Drittels der Ausbildung & 76,3 & 1130 \\
\hline Wahl der Ausbildungsstätte nach Ruf & $2,33(1,17)$ & 1060 \\
\hline Wahl der Ausbildungsstätte aus beruflichen Gründen & $2,67(1,36)$ & 1062 \\
\hline \multicolumn{3}{|l|}{ Gruppe 3: strukturell-prozessuale Parameter der Ausbildungsstätte } \\
\hline Anteil der Seminare/Arbeit in Kleingruppen & $84,94(27,81)$ & 1159 \\
\hline Größe der Supervisionsgruppen/Klassen & $3,96(1,66)$ & 1063 \\
\hline $\begin{array}{l}\text { Analyse der Videosequenzen während der Supervision (Anteil der Auszubildenden } \\
\text { mit Videosupervision) }\end{array}$ & 61,9 & 1044 \\
\hline Pädagogische Qualifikation und didaktische Fähigkeiten der Supervisoren & $1,93(1,84)$ & 1003 \\
\hline Angemessene Supervisionsintensität & $2,18(1,09)$ & 986 \\
\hline $\begin{array}{l}\text { Persönliche Unterstützung durch Supervisoren zur Verbesserung des professionellen } \\
\text { therapeutischen Verhaltens }\end{array}$ & $1,97(1,97)$ & 976 \\
\hline Gesamtkosten der Ausbildung, Euro & $17046,72(6163,04)$ & 1081 \\
\hline Organisation der Ausbildung & $2,52(1,17)$ & 1153 \\
\hline Kosten/Leistungs-Verhältnis & $2,72(1,10)$ & 1142 \\
\hline Beratung und Begleitung durch die Institutsleiter während/am Ende der Ausbildung & $2,78(1,23)$ & 1120 \\
\hline Unterstützung bei Schwierigkeiten während der Ausbildung durch die Institutsleiter & $2,73(1,29)$ & 1000 \\
\hline Berücksichtigung der Bedürfnisse der Auszubildenden & $3,05(1,21)$ & 1080 \\
\hline Interesse der Institutsleiter an Feedback von den Auszubildenden & $2,50(1,29)$ & 1079 \\
\hline Konsequenzen der Evaluierungsergebnisse für zukünftige Seminare/Ausbildungen & $2,81(1,40)$ & 895 \\
\hline
\end{tabular}

Tabelle 4 zeigt angepasste Beta-Gewichte, um den relativen Beitrag der einzelnen Variablen zu den Kompetenzerwartungen festzustellen. Zwischen 4 und 6 Prädiktoren mit signifikanten Beta-Gewichten konnten für jedes Kriterium identifiziert werden.

Für alle 5 Bereiche der Kompetenzerwartungen waren das Kosten/Leistungs-Verhältnis und die Unterstützung durch die Ausbilder signifikante Prädiktoren. Die stärksten Prädiktoren, die in 2 oder 3 der 5 Kompetenzbereiche wirkten, waren die geschätzte Dauer der Ausbildung und die Art der Ausbildung (Erwachsenen- oder Kinder- und Jugendlichenpsychotherapie-Ausbildung).

Die Prädiktoren, die ein signifikantes Beta-Gewicht für einen der Kompetenzbereiche zeigten, waren die Gesamtkosten der Ausbildung für das Kriterium Selbsterfahrung, die Häufigkeit der Supervision für das Kriterium Formalitäten und die pädagogische/didaktische Qualifikation der Ausbilder für das Kriterium Wissen zu Symptomen/Ätiologie. Alle anderen Prädiktoren trugen nicht signifikant zur Varianzerklärung bei.

\section{Diskussion}

Theoretisch begründet wählten wir 3 Gruppen verschiedener Variablen als Prädiktoren für die Kompetenzerwartungen aus: soziodemografische Parameter, allgemeine Ausbildungsbedingungen (z.B. Dauer der Ausbildung und Ruf der Ausbildungsstätte) sowie strukturell-prozessuale Parameter (z.B. Kursgröße, Supervisionsintensität). Die erklärte Varianz des Kriteriums (Kompetenzbewertung) umfasste aufgrund der Prädiktoren 9-12\%. Alle Prädiktoren, die signifikant in allen 5 Bereichen der Kompetenzerwartungen waren, gehörten zur Gruppe 3 (strukturell-prozessuale Parameter). Nur der Parameter «geschätzte Ausbildungsdauer» der Gruppe 2 (allgemeine Bedingungen) war signifikant in 3 der 5 Bereiche der Kompetenzerwartungen. Wie erwartet, hängt eine länger geschätzte Ausbildungsdauer mit geringeren Kompetenzbewertungen zusammen.

Die 2 Prädiktoren aus Gruppe 3 (strukturell-prozessuale Parameter) «Kosten/Leistungs-Verhältnis» und «Unterstützung durch den Ausbilder» waren besonders bemerkenswert, da sie signifikante Beta-Gewichte in allen 5 Kompetenzberei- 


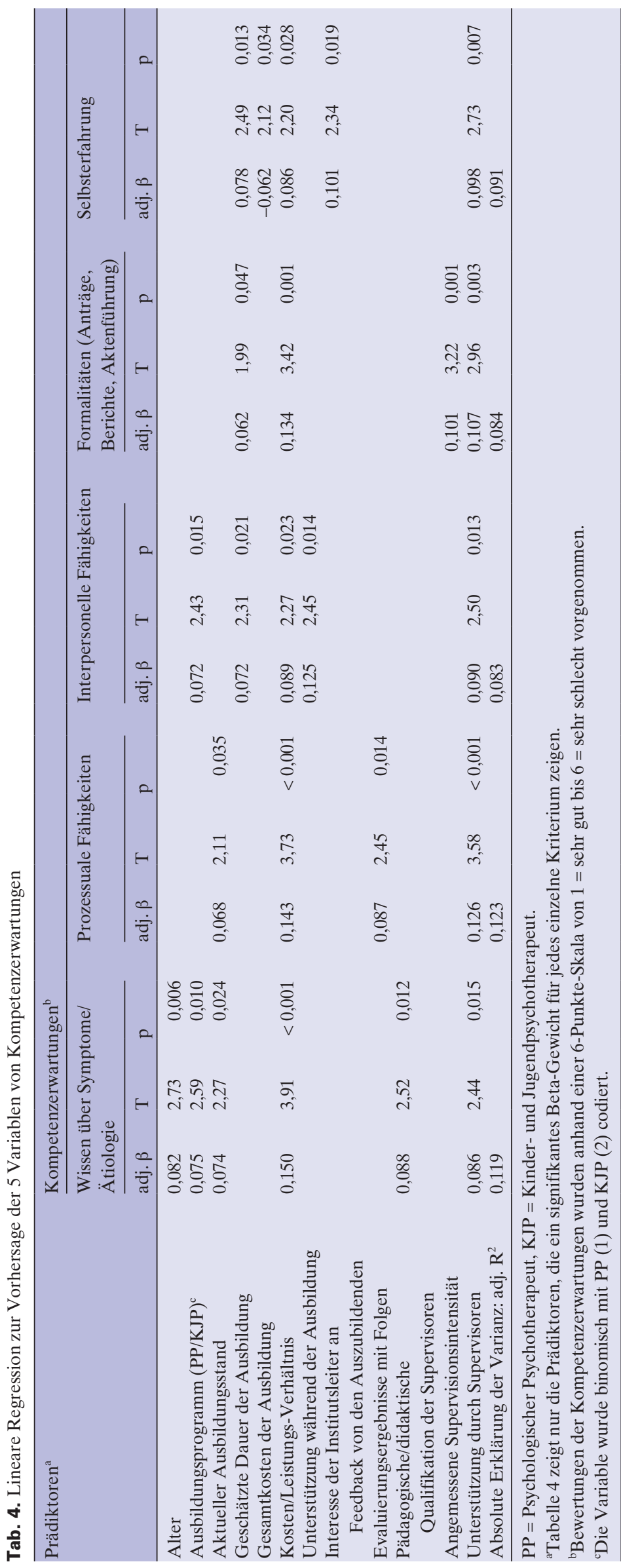

chen zeigten. Ein als gut bewertetes Kosten/LeistungsVerhältnis der Ausbildung im Sinne von «Das Geld hat sich gelohnt» führt zu höheren Erwartungen im Hinblick auf einen angemessenen Kompetenzgewinn. Man könnte das Kosten/Leistungs-Verhältnis auch als globalen Indikator für «Kundenzufriedenheit» mit der Ausbildung interpretieren. Zufriedenheit im Sinne des Modells von Orlinsky und Roennestad könnte zum Erlebnis von «Currently Experienced Growth» beitragen. In ähnlicher Weise reflektiert der hoch bewertete Prädiktor «Unterstützung durch den Supervisor» eine intensive Betreuung des Ausbildungskandidaten im Gewinnen von professioneller Kompetenz als Psychotherapeut. Die Ausbildungsund Prüfungsverordnung für Psychotherapeuten fordern ein Minimum von 150 Supervisionsstunden mit mindestens 50 Stunden Einzelsupervision. Somit wird deutlich, dass «training on the job» sehr bedeutsam für die Entwicklung positiver Kompetenzerwartungen ist.

Ein Blick auf die zahlreichen potenziellen Prädiktoren, die sich dann empirisch als nicht bedeutsam erwiesen, legt interessante Schlussfolgerungen nahe. Die erwarteten Berufskompetenzen erwiesen sich als unabhängig von Alter, Geschlecht und eher allgemeinen Ausbildungsbedingungen.

Zusammengefasst zeigten sich aber 2 Parameter, die einen Teil der Kompetenzerwartungen vorhersagen konnten: erstens ein globaler Kundenzufriedenheitsparameter inklusive der Finanzinvestition und Qualität der Ausbildung (Kosten/Leistungs-Verhältnis) und zweitens ein interpersoneller Parameter inklusive der Erfahrung von persönlicher Unterstützung während der Supervision.

\section{Grenzen/Einschränkungen}

Alle gesammelten Daten sind Selbstauskünfte von Auszubildenden. Somit gibt es nur eine Quelle, aus der die Daten stammen. Weiterhin sind Selbstauskünfte und Eigenbewertungen immer anfällig für diverse Einflüsse, wie z.B. soziale Erwünschtheit, Selbstwertsicherung und fundamentale Attributionsfehler. Es ist auch unbekannt, wie stark die Kompetenzerwartungen mit den wirklichen, in der Ausbildung gewonnenen Kompetenzen korrelieren. Weiterhin war die Gruppe der Befragten nicht homogen, d.h. die einzelnen Auszubildenden wurden in unterschiedlichen Ausbildungsphasen befragt.

Die erklärte Varianz des Gesamtmodells ist gering. Somit war die Vorhersage der Kompetenzerwartungen durch distale Parameter der Auszubildenden, der Ausbildung und der Institutionen nur zum Teil erfolgreich. Neben den angeführten möglichen inhaltlichen Begründungen könnten auch methodologische Aspekte dieses Ergebnis erklären. Die vorhergesagten Kompetenzerwartungen in allen 5 Bereichen hatten eine Varianz mittlerer Ausprägung und bildeten inhaltlich bedeutsame Differenzen ab. Aber die Ausbildungskandidaten schöpften nicht 
die ganze 6-Punkte-Skala aus. Ein solcher Befund ist von anderen Studien im Bereich der Patienten- oder Kundenzufriedenheit in der Gesundheitsvorsorge [z.B. Williams et al., 1998] bekannt. Unter anderem kann dies mit sozial-psychologischen Prozessen (z.B. dissonanztheoretischen Prozessen) erklärt werden. Aus einer methodologischen Sicht könnte daher auch die im mittleren Bereich liegende Varianz verantwortlich für die geringere erklärte Varianz in den Regressionsmodellen sein.

\section{Vorschläge für die Ausbildung und zukünftige Forschung}

Nach der Datenlage könnte man zu der Schlussfolgerung kommen, dass eine effizientere und kürzere Ausbildung zu höheren Kompetenzerwartungen führt. Die Auszubildenden bräuchten mehr finanzielle Unterstützung, um ihre Ausbildung zügig abschließen zu können, ohne auf zusätzliche Arbeit zum Lebenserwerb angewiesen zu sein. [Glaesmer et al., 2009]

Die einzelnen institutsspezifischen strukturellen Parameter scheinen weniger wichtig für die Ausbildungskandidaten $\mathrm{zu}$ sein. Bedeutsam ist jedoch eine subjektiv erlebte Angemessenheit des Kosten/Leistungs-Verhältnisses des jeweiligen Ausbildungsangebotes. Das unterstreicht die Sinnhaftigkeit verschiedener Ausbildungsangebote innerhalb der gesetzlichen Rahmenbedingungen, die sich in der Schwerpunktsetzung, der organisatorischen Qualität und der Kostenstruktur unterscheiden können. Durch eine so angebotene Vielfalt kann vermutlich den unterschiedlichen Bedürfnissen der Ausbildungskandidaten am besten Rechnung getragen werden.

Aus empirischer Perspektive scheinen die Erfahrungen mit persönlichen, professionellen Vorbildern entscheidend wichtig für den auf Kommunikation basierenden Beruf des Psy- chotherapeuten zu sein. Die während der Ausbildung erfahrene Unterstützung durch einen kompetenten Supervisor, vermutlich durchaus mit Anklängen an eine traditionelle Lehrer-Schüler-Beziehung, scheint eine wichtige Quelle der positiven Selbsteinschätzung hinsichtlich der eigenen Berufskompetenzen zu sein.

Insgesamt zeigen die Ergebnisse die Wichtigkeit von personenbezogenen Erfahrungen für die Entwicklung beruflicher Kompetenzerwartungen in der psychotherapeutischen (VT-) Ausbildung. Die weitere Forschung sollte diese Fragen ausführlicher untersuchen, z.B. im Rahmen von LängsschnittStudiendesigns. Erst wenn wir besser verstehen, wie man ein «guter» Therapeut wird, können wir professionelle Lehr- und Lernprozesse zielführend fördern und verbessern.

Psychotherapeut zu sein, ist Belohnung und Herausforderung zugleich. Im Allgemeinen scheinen deutsche Psychotherapie-Ausbildungskandidaten zufrieden mit ihrer Ausbildung und beruflichen Entwicklung zu sein [Sonntag et al., 2009]. Dennoch ist es eine Herausforderung, mehr und genaueres Wissen darüber zu generieren, was benötigt wird, um Therapeuten auszubilden, die wirklich gut und erfolgreich mit ihren Patienten und auch mit sich selbst umgehen können. Daher gibt es zu diesem Thema mit Sicherheit weiteren Forschungsbedarf.

\section{Disclosure Statement}

Der Studie liegen Daten zugrunde, die im Rahmen einer DVT-internen und von den Ausbildungsinstituten selbst finanzierten Evaluationsstudie erhoben wurden. G.Z. und W.S. sind Leiter von DVT-Ausbildungsinstituten.

\section{Literatur}

Berghoff S, Federkeil G, Giebisch P, Hachmeister C-D, Hennings M, Müller-Böling D: CHE-Hochschulranking. Vorgehensweise und Indikatoren. Arbeitspapier 88. Gütersloh, Centrum für Hochschulentwicklung, 2007.

Cohen J: Statistical Power Analysis for the Behavioral Sciences, ed 2. Hillsdale, Lawrence Erlbaum, 1998.

Glaesmer H, Sonntag A, Barnow S, Brähler E, Fegert JM: Psychotherapeutenausbildung aus Sicht der Absolventen. Ergebnisse der Absolventenbefragung im Rahmen des Forschungsgutachtens. Psychotherapeut 2009;54:437-444.

Grawe, K: Psychotherapie-Ausbildung: Gemeinsamkeiten statt Grenzen betonen. Psychoscope 1994; 15:4-6.

Laireiter AR, Botermann JF: Ausbildungsforschung in der Psychotherapie; in Laireiter AR, Willutzki U (Hrsg): Ausbildung in Verhaltenstherapie. Göttingen, Hogrefe, 2005, pp 53-101.

Orlinsky DE, Roennestad MH: How Psychotherapists Develop. A Study of Therapeutic Work and Professional Growth. Washington, APA, 2005.
Scherer U, Mayer K, Neuser J: Die schriftlichen Prüfungen nach dem Psychotherapeutengesetz: Ergebnisse und Analysen. Psychotherapeutenjournal 2005;3:212-221.

Schulz H, Barghaan D, Harfst T, Koch U: Gesundheitsberichterstattung des Bundes. Psychotherapeutische Versorgung, 2008.

Schulz W, Flotho B, Lübke A, Hahlweg K: Dokumentation und Evaluation des Weiterbildungsganges Braunschweig in Klinischer Psychologie/Psychotherapie [Documentation and Evaluation of the Advanced Training Course in Clinical Psychology/Psychotherapy at Brunswick]. Verhaltenstherapie 1998:8:234-244.

Sonntag A, Glaesmer H, Barnow S, Brähler E, Fegert JM, et al.: Die Psychotherapeutenausbildung aus Sicht der Teilnehmer. Ergebnisse einer Ausbildungsteilnehmerbefragung im Rahmen des Forschungsgutachtens. Psychotherapeut 2009;54:427-436.

Strauß B, Freyberger HJ: Ausbildungsforschung in der Psychotherapie. Ergebnisse des Forschungsgutachtens. Psychotherapeut 2009;54:409-410.

Strauß B, Kohl S: Themen der Ausbildungsforschung in der Psychotherapie [Themes of training research in psychotherapy]. Psychotherapeut 2009;54:411-426. von Hölzel HH: Zur finanziellen Situation der Psychotherapeuten in Ausbildung: Ergebnisse einer internetgestützten Fragebogenstudie. Psychotherapeut 2006;3:232-237.

von Hopffgarten von Laer A, Mempel S: Evaluation der verhaltenstherapeutischen Ausbildung am Institut für Fort- und Weiterbildung in klinischer Verhaltenstherapie (IFKV), Bad Dürkheim. Verhaltenstherapie 1998;8:259-262.

Williams B, Coyle J, Healy D: The meaning of patient satisfaction: An explanation of high reported levels. Soc Sci Med 1998;47:1351-1359.

Willutzki U, Botermans J-F; SPR Collaborative Research Network: Ausbildung in Psychotherapie in Deutschland und der Schweiz und ihre Bedeutung für die therapeutische Kompetenz [Training in psychotherapy in Germany and Swizerland and its relevance for therapeutic competency]. Psychotherapeut 1997;42:282-289.

Zimmer D, Zimmer FT: Wie hilfreich sind die Bausteine einer Verhaltenstherapie-Weiterbildung. Verhaltenstherapie 1998;8:254-257. 